\title{
Summation: Assessment of a configural theory
}

\author{
ROBERT A. RESCORLA \\ University of Pennsylvania, Philadelphia, Pennsylvania
}

\begin{abstract}
In five experiments, rats were given Pavlovian pairings of auditory and visual stimuli with delivery of food pellets. Experiment 1 found greater responding to an $A B$ compound after training with the individual $A$ and $B$ stimuli, compared with responding both to the $A$ and $B$ elements and to a separately trained $\mathrm{CD}$ compound. Experiment 2 found this enhanced responding to depend on the associative strengths of $A$ and $B$. In Experiment 3, responding was greater to a CD compound than to the other compounds after an $\mathrm{AB}-, \mathrm{AD}+, \mathrm{BC}+$ training procedure. In Experiment 4, responding to an $\mathrm{AB}$ compound was greater than that to the elements after $\mathrm{A}$ was reinforced on a $100 \%$ schedule and $\mathrm{B}$ on a 50\% schedule. In Experiment 5, responding to an $\mathrm{AC}$ compound was greater than that to either $\mathrm{A}$ or $\mathrm{C}$ after an $\mathrm{AB}+, \mathrm{CD}+, \mathrm{A}-$ training procedure. A configural theory, such as that proposed by Pearce (1987), anticipates summation in none of these procedures, unless the conditioned context is assumed to have a salience greater than zero. In order to predict summation in Experiments 3, 4, and 5, a context salience greater than that of the elements must be assumed. However, such an assumption also anticipates that extinction of a $100 \%$ stimulus should eliminate responding to a $50 \%$ stimulus. The results of Experiment 3 contradicted that prediction. These results conform better to the expectations of elemental models of conditioning.
\end{abstract}

A problem of continuing interest in associative learning is that of specifying the behavior to a compound stimulus, $A B$, in terms of the behavior to its components, $A$ and $B$. One can identify two broad classes of theories that attempt to deal with this problem.

What has sometimes been call the "elemental" approach focuses on the compound as composed of its constituent elements. According to this approach, the compound stimulus consists primarily of its component parts and the associative strength of the compound is derived by some rule for summing the associative strengths of those components. This historically popular view has been incorporated into many theories of learning (e.g., Hull, 1943; Rescorla \& Wagner, 1972).

A less popular, but attractive, approach has been called the "configural" approach. This approach views each compound as a new stimulus, distinct from its elements. Responding to that compound then depends on its receiving generalization based on its similarity to the trained elements. Although historical versions of this approach have sometimes left that similarity poorly defined, a recently proposed configural theory specifies the element/compound relation in terms of an explicit generalization rule linking them (Pearce, 1987, 1994).

Each of these approaches has its attractions. The elemental approach offers a simple, analytic basis for building up a compound from its component parts. It can provide a natural account of many phenomena that result from compound presentation, such as summation, blocking, and over-

This research was supported by National Science Foundation Grant BNS-88-03514. Correspondence should be addressed to R. A. Rescorla, Department of Psychology, University of Pennsylvania, 3815 Walnut Street, Philadelphia, PA 19104 (e-mail: rescorla@psych.upenn. edu). shadowing. On the other hand, it is less natural for some instances in which responding to the compound seems quite different from what might be expected on the basis of responding to the elements. For instance, it is troubled by negative patterning in which the animal learns to respond to the $A$ and $B$ elements when presented separately but not when presented together in compound. Such phenomena have led to the elaboration of elemental theories so as to include configural-like components, such as the "unique cue" that Wagner and Rescorla (1972) envisioned as contributing to a compound. By contrast, the configural approach described by Pearce (1987) deals with such patterning discriminations gracefully, but it is frequently less helpful in understanding other phenomena, such as summation.

The present experiments focus on the prediction of summation from configural theories. In a typical summation procedure, the A and B stimuli are separately conditioned, and then responding is examined to their joint presentation, $\mathrm{AB}$. In a wide variety of conditioning preparations (see, e.g., Forbes \& Holland, 1985; Kehoe, 1986; Pavlov, 1927; Reberg, 1972), responding to the AB compound is more substantial than that to the elements. Within an elemental context, this result is interpreted as the compound's having a higher associative strength than either of its elements alone, by virtue of some combination rule that adds those strengths. However, there are some instances in which summation does not seem so readily observed. For instance, both Aydin and Pearce (1995) and Rescorla and Coldwell (1995) have recently reported difficulty observing summation with localized visual components in an autoshaping preparation with pigeons. That result seems difficult for an elemental theory but more congenial to a configural account.

In the present experiments, summation was studied in a preparation in which rats approach a food magazine dur- 
ing a signal of food delivery. Experiments 1 and 2 documented the occurrence of summation in that preparation, using a fairly demanding set of criteria. Experiments 3 , 4 , and 5 explored one possible way that a configural theory might incorporate that finding, by appealing to the contribution of background stimuli.

\section{EXPERIMENT 1}

The goal of this experiment was to document the occurrence of summation in a magazine approach preparation. The inference that summation has been obtained is commonly made whenever the responding to an $A B$ test compound is greater than that to the A and $B$ trained elements. However, there are several alternative ways that this result might come about, even in the absence of the $\mathrm{AB}$ compound's having higher associative strength than its elements. The present experiment took care to study summation under conditions that allow evaluation of two of those alternatives.

First, one can anticipate that even though $\mathrm{A}$ and $\mathrm{B}$ receive the same treatment, there will be differences in their associative strengths. Moreover, those differences may well be distributed differently across the stimuli for different subjects of an experiment, such that A may be stronger than $B$ for some subjects whereas $B$ is stronger than $A$ for others. It is then possible that when the $A B$ compound is presented, each subject simply responds on the basis of the stronger of the two elements in the compound. Responding during the compound might then be greater than the average responding to the A or B elements, even though in fact there is no actual combination of the associative strengths. That is, a performance rule in which responding was controlled exclusively by the stronger of the two elements would yield apparent summation. In order to evaluate that possibility, the present experiment compared the responding to $\mathrm{AB}$ with whichever of the $\mathrm{A}$ and $B$ stimuli showed greater responding, determined for each individual animal.

Second, the fact that the $\mathrm{AB}$ compound is composed of two elements implies that its total sensory stimulation of the animal is greater than that achieved by the A and B elements individually. It is possible that the greater responding to the compound reflects that difference in total sensory stimulation (Whitlow \& Wagner, 1972). To evaluate that possibility, the present experiment compared responding during $\mathrm{AB}$ not only to that during $\mathrm{A}$ and $\mathrm{B}$ but also to that during another compound, $\mathrm{CD}$, which had itself been paired with the food. The level of stimulation from $C D$ should be comparable to that from $\mathrm{AB}$, but current elemental theories anticipate that overshadowing should guarantee that the $\mathrm{CD}$ has an associative strength less than that controlled by the $\mathrm{AB}$ compound.

\section{Method}

\section{Subjects and Apparatus}

The subjects were 16 male Sprague-Dawley rats about 90 days old. They were housed in individual cages and maintained on a food deprivation regime that kept them at $80 \%$ of their ad-lib body weight. They had free access to water in the home cage.

The apparatus consisted of four operant chambers measuring $22.9 \times 20.3 \times 20.3 \mathrm{~cm}$, identical to those used in previous reports (e.g., Colwill \& Rescorla, 1985). The two end walls of each chamber were aluminum; the side walls and ceiling were clear Plexiglas. The floor of the chamber was composed of $0.48-\mathrm{cm}$ stainless steel rods, spaced $1.9 \mathrm{~cm}$ apart. Each chamber had a recessed food magazine in the center of one end wall. Two small metal cups measuring $1.25 \mathrm{~cm}$ in diameter and $1.5 \mathrm{~cm}$ deep were sunk side by side in the floor of each food magazine. An infrared detector and emitter system were mounted on the side walls of the magazine, permitting automatic recording of head movements into the magazine.

Each chamber was enclosed in a sound-and light-resistant shell. Mounted on the inside wall of this shell were speakers that permitted the presentation of a white noise and an $1800-\mathrm{Hz}$ tone, each measuring approximately $76 \mathrm{~dB}$ re $20 \mu \mathrm{N} / \mathrm{m}^{2}$ against a background level of $62 \mathrm{~dB}$. Also mounted on that wall was a $6-W$ bulb that could be illuminated to provide a light stimulus during the otherwise dark session. Another 6-W light was mounted on the center of the ceiling of the chamber; this light could be flashed at a rate of $1 / \mathrm{sec}$ to provide a flashing stimulus. The outside ceiling of the shell supported two solenoid-operated gravity feed valves that were connected via plastic tubing to the cups in the food magazine. One system permitted the presentation of $.3 \mathrm{ml}$ of an $8 \%$ sucrose solution; the other permitted the presentation of $.3 \mathrm{ml}$ of a $15 \%$ Polycose solution. Neither of these devices was used in the present experiments. Also attached to that food magazine was a dispenser containing 45-mg pellets (P. J. Noyes, Formula A).

Experimental events were controlled and recorded automatically by relays and microprocessors located in an adjoining room.

\section{Procedure}

Magazine training. On the 1 st day, the animals received a 20 min magazine training session, during which 20 noncontingent deliveries of pellets were given, at time intervals variable around a mean of $1 \mathrm{~min}$.

Pavlovian conditioning. On each of the next 14 days, all animals received Pavlovian conditioning of two elements (A and $B$ ) and one compound (CD). Each session contained eight 30 -sec presentations of each stimulus, each ending in a pellet. The $A$ and $C$ stimuli were identified with the steady light and flashing light; the $\mathrm{B}$ and $\mathrm{D}$ stimuli were the noise and tone. Stimulus identification was such that each of the four possible auditory-visual compounds was used as $C D$ in a quarter of the animals, with the remaining stimuli playing the roles of $A$ and $B$. The intertrial interval (ITI), measured between stimulus initiations, was variable around a mean of $120 \mathrm{sec}$. The number of head entries into the magazine was recorded during each $30-\mathrm{sec}$ conditioned stimulus (CS) and during the 30-sec stimulus-free period preceding each CS.

Test. On the next day, the animals received Pavlovian conditioning in the same manner, except that during the last half of the session four reinforced presentations of the $A B$ compound were intermixed with the four training trials of each type. It was responding to the $\mathrm{AB}$ compound, compared with that to the $\mathrm{CD}$ compound and to the $\mathrm{A}$ and $\mathrm{B}$ elements, that was of most interest during this period.

\section{Results and Discussion}

Pavlovian conditioning proceeded smoothly to both the $\mathrm{A}$ and $\mathrm{B}$ elements and the CD compound. On the final day, the mean magazine entries per minute were 6.0 , $13.2,14.0$, and 13.4 during the pretrial period, the auditory and visual elements, and the CD compound, respectively. All stimuli elicited greater responding than that ob- 


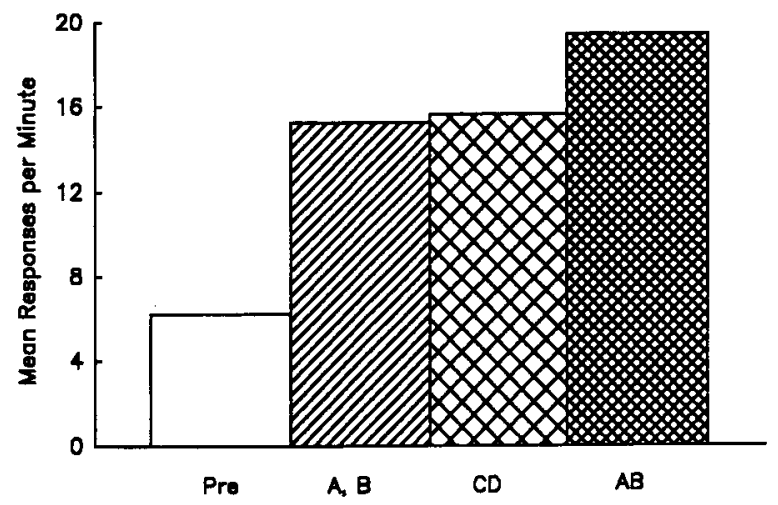

Figure 1. Results of the test session of Experiment 1. Mean number of magazine entries is shown during a 30-sec period prior to any stimulus, during the separately trained $A$ and $B$ stimuli, during the trained $C D$ compound, and during the tested $A B$ compound.

served during the pre-CS period [Wilcoxon $T \mathrm{~s}(16)=0$, $p$ s $<.01]$.

The data of most interest, from the test session, are shown in Figure 1. That figure displays responding prior to the $\mathrm{CS}$, during the average of the $\mathrm{A}$ and $\mathrm{B}$ elements, during the trained $\mathrm{CD}$ compound, and during the tested $\mathrm{AB}$ compound. During that test, all stimuli continued to elevate responding relative to the pre-CS period. But responding during the $\mathrm{AB}$ compound was greater than that during either the CD compound $[T(15)=2, p<.01]$ or the average of that during the $A$ and $B$ elements $[T(16)=0$, $p<.01]$. Most impressively, responding during the $\mathrm{AB}$ compound was also greater than that to whichever of the $\mathrm{A}$ and $\mathrm{B}$ elements showed greater responding, determined un an individual rat basis $[T(16)=23.5, p<.02]$.

These data show clear evidence of substantial summation in a magazine approach preparation. The comparison with the CD compound and the maximum of the $A$ and $B$ elements suggests that neither stimulus intensity nor individual sampling of the stronger of the two elements can account for the results. They therefore suggest that there was a true summation of the associative strengths of the elements.

\section{EXPERIMENT 2}

If associative strengths of separately trained elements do summate when they are presented in compound, then one would expect that the magnitude of the response to the compound would depend on the associative strengths of the individual elements. Although Experiment 1 found responding to the tested $\mathrm{AB}$ compound to exceed that both to the A and $\mathrm{B}$ elements and to the trained $\mathrm{CD}$ compound, it does not provide evidence that this summation depends on the strengths of the $A$ and $B$ elements. This is of some importance because there are mechanisms other than summation that might result in responding to the $\mathrm{AB}$ compound that exceeds that to the elements. For instance, Aydin and Pearce (1995) have argued that with a relatively long $\mathrm{A}$, inhibition of delay might develop to the early portion of the stimulus. Under those conditions, presenting even a neutral B in conjunction with A might lead to enhanced responding, because of disinhibition. They found evidence that at least one apparent instance of summation in an autoshaping procedure with birds could be so interpreted.

Consequently, in this experiment summation was assessed under circumstances in which a reinforced A stimulus was presented in compound both with a reinforced $B$ and with a nonreinforced $C$. The expectation was that summation of associative strengths would yield more substantial responding to the $\mathrm{AB}$ compound than either the $\mathrm{A}$ and $\mathrm{B}$ elements or the $\mathrm{AC}$ compound.

\section{Method}

\section{Subjects and Apparatus}

The apparatus was the same as that of Experiment 1 . The subjects were 16 rats of the same origin and maintained in the same manner as those of Experiment 1.

\section{Procedure}

Pavlovian conditioning. The animals were magazine trained with pellets in the manner of Experiment 1 . On each of the next 14 days they received Pavlovian conditioning in the manner of Experiment 1 . Each session contained eight 30 -sec presentations each of $\mathrm{A}+, \mathrm{B}+$, and $\mathrm{C}-$. For half the animals, $\mathrm{A}$ was the noise and $\mathrm{B}$ and $\mathrm{C}$ were counterbalanced as the flashing and steady light. For the other half of the animals, $A$ was the steady light and $B$ and $C$ were counterbalanced as the tone and noise. In other regards, the acquisition procedure was identical to that of Experiment 1.

Test. On the next day, the animals received Pavlovian conditioning in the same manner except that during the last half of the session, two reinforced presentations each of $\mathrm{AB}$ and $\mathrm{AC}$ were intermixed with the four training trials of each type.

\section{Results}

Pavlovian conditioning proceeded without incident. On the final day, the mean magazine entries per minute were $4.8,16.8,14.8$, and 6.0 during the pretrial period, $\mathrm{A}$, $\mathrm{B}$, and $\mathrm{C}$, respectively. Responding during $\mathrm{A}$ and $\mathrm{B}$ exceeded that during the pretrial and $C$ periods $[T \mathrm{~s}(16)=$ $0, p s<.01]$.

The data of primary interest, from the second half of the session during which $\mathrm{AB}$ and $\mathrm{AC}$ were tested, are shown in Figure 2. That figure displays the mean magazine entries per minute during the prestimulus period, during the $\mathrm{A}, \mathrm{B}$, and $\mathrm{C}$ elements, and during the $\mathrm{AB}$ and $\mathrm{AC}$ compounds. It is clear that the reinforced elements continued to elicit substantial responding, whereas the nonreinforced $\mathrm{C}$ element did not. Of more interest, responding to the combination of the two reinforced elements $(A B)$ was substantial, whereas responding to the combination of a reinforced and nonreinforced element $(\mathrm{AC})$ was similar to that of the reinforced element alone. Statistically, responding to the $\mathrm{AB}$ compound exceeded that both to the maximum of the $\mathrm{A}$ and $\mathrm{B}$ elements $[T(15)=24, p<.05]$ and to the $\mathrm{AC}$ compound $[T(16)=26, p<.05]$.

A more detailed inspection of the distribution of responding during the stimuli suggested that responding grew with each $10-\sec$ period, a pattern consistent with 


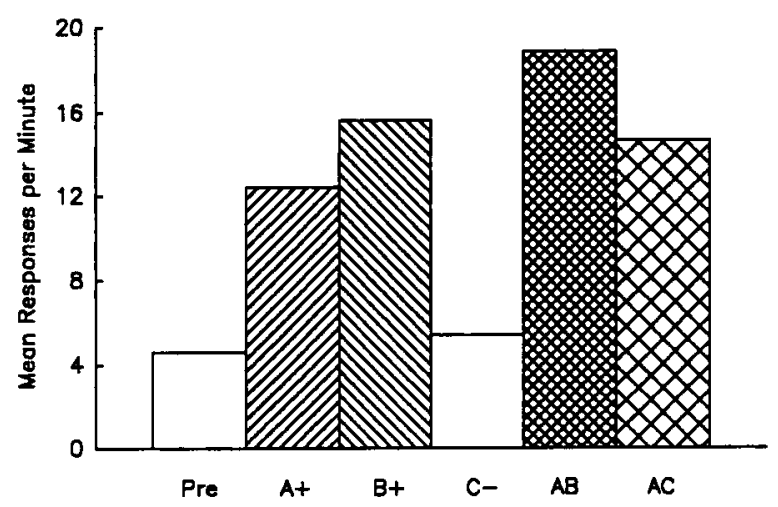

Figure 2. Results of the test session of Experiment 2. Responding is shown during the 30-sec period prior to any stimulus, during the trained $\mathrm{A}+, \mathrm{B}+$, and $\mathrm{C}-$ elements, and during the tested $\mathrm{AB}+$ and $\mathrm{AC}+$ compounds.

inhibition of delay. The mean rates of responding in successive $10-\mathrm{sec}$ periods to the A and B stimuli were 9.2 , 14.0, and 18.7. However, those to $A B$ were $15.9,19.1$, and 21.4, whereas those to AC were 11.8, 12.4, and 19.9. Although the short time intervals and small number of test trials yielded a high level of variability, these results suggest that the summation to $\mathrm{AB}$ appeared at each time interval. Summation was numerically somewhat smaller at the end of the stimulus, presumably due to ceiling effects; but its appearance throughout the stimulus seems inconsistent with a disinhibition account.

\section{Discussion}

These results agree with those of Experiment 1 in showing greater responding to a compound of two separately trained elements than to the elements themselves. Moreover, they show that this result depends on the associative strength of the elements in a manner expected on the basis of summation.

The results of Experiments 1 and 2 are natural from the point of view of an elemental theory. But they are less straightforward for a configural view of the $A B$ compound. In fact, the best specified configural theory (Pearce, 1987) appears not to anticipate summation of this sort. According to that theory, responding to the AB compound is based on the generalization that it receives from the A and B elements. That generalization is specified in terms of a rule dependent on the sensory potency (p) of the elements that $A B$ shares with $A$ and $B$. In particular, the generalization that $\mathrm{AB}$ receives from $\mathrm{A}$ is determined by the proportion of each stimulus constituted by the shared A element. These proportions are multiplied by each other and by the associative strength of the shared $A, v(A)$. That is, the generalization that $A B$ receives from $A$ is $v(A) * p(A) /[p(A)+p(B)] *[p(A) / p(A)]$. $A$ comparable expression governs the generalization to $A B$ from $B$. The total generalized strength of $A B$ derived from both $A$ and $B$ is thus $v(A) * p(A) /[p(A)+p(B)]$ plus $v(B) * p(B) /[(p(A)+p(B)]$, or a weighted average of the strengths of $A$ and $B$. That in turn means that the strength of the $A B$ compound should lie somewhere between that of $\mathrm{A}$ and $\mathrm{B}$, rather than exceeding them both.

Since the present results clearly contradict this simple application of a configural theory, it is natural to look for some way in which this theory might be elaborated. One approach would be to modify the generalization rule so that a more substantial portion of the elements' strength generalized to the $\mathrm{AB}$ compound. Effectively, elemental theories are the limiting case of such a modification, since they envision a rule with complete generalization. However, a less drastic modification of the configural theory is suggested by the role that many theories give to background stimuli. If one acknowledges the presence of a background stimulus $(\mathrm{X})$ throughout the experiment, then a summation experiment could be characterized as training $\mathrm{AX}$ and $\mathrm{BX}$, mixed with extinction of $\mathrm{X}$ alone, followed by testing of $A B X$. Under these circumstances, this model predicts summation to ABX. The reason for this is that the excitatory conditioning of $\mathrm{AX}$ and $\mathrm{BX}$ leads to generalization of excitation to the $\mathrm{X}$ background. According to the Pearce (1987) model, when that background is then extinguished, it develops inhibition, which in turn generalizes back to AX and BX. However, AX and BX continue to be reinforced, and therefore to develop a total net associative strength approximating 1 . The inhibitory generalization from $X$ encourages them to develop yet more substantial excitation of their own. When ABX is tested, it receives generalization of this more substantial excitation twice, once from $\mathrm{AX}$ and once from $\mathrm{BX}$, but it receives generalization of the inhibition from $X$ only once. More formally, one can show that under these conditions, the associative strength of $\mathrm{ABX}$ becomes 1 $+\mathrm{p}(\mathrm{X}) /[\mathrm{p}(\mathrm{A})+\mathrm{p}(\mathrm{B})+\mathrm{p}(\mathrm{X})]$, where 1 is the asymptote that the unconditioned stimulus (US) is capable of producing and $p$ represents the potency of each stimulus. From this expression, it is clear that if the potency of the background, $p(X)$, is zero, then the associative strength of $\mathrm{ABX}$ is 1 , the same as that of $\mathrm{AX}$ and $\mathrm{BX}$. However, the greater $\mathrm{p}(\mathrm{X})$, the more the strength of the ABX compound will exceed 1 , and the more summation will be observed. Consequently, assuming a contribution of the background to performance allows this theory to anticipate summation.

Of course, it is difficult to evaluate the proposition that the background is potent. Although it has been common to view the background as less potent than the discrete stimuli themselves (see, e.g., Rescorla \& Wagner, 1972), there is little experimental basis for making that assumption. The evaluation of background potency is made particularly difficult because one natural assessment of the contribution of the background, testing in another background, does not yield straightforward implications for the issue at hand. The performance observed in a second background depends not only on the potency of the backgrounds but also on the degree to which they generalize to each other. Consequently, the failure of a contextual shift to have a substantial effect may not indicate 
that the context was ineffective but only that the training and testing context are viewed by the animal as similar to each other.

Consequently, the remaining experiments adopted a somewhat different strategy for evaluating the success of this elaboration of the configural model. They examined situations in which summation would not be anticipated by this model even assuming a contribution of a moderately potent context. Experiment 4 also examined further experimental consequences of assuming that the context was sufficiently salient to generate the summation that it observed.

\section{EXPERIMENT 3}

The intention of this experiment was to examine the possibility of summation under circumstances where an elemental theory makes a positive prediction but the Pearce configural theory does not, even with the assumption that the background plays a role equal to that of the discrete stimuli. The procedure employed involved four stimuli, presented in three compounds: $\mathrm{BC}+, \mathrm{AD}+$, and $\mathrm{AB}-$.

According to most elemental theories (e.g., the Rescorla-Wagner model), this conditioning should result in high levels of responding to $\mathrm{AD}$ and $\mathrm{BC}$ but low levels to $\mathrm{AB}$. This should be accomplished by the individual $\mathrm{C}$ and $D$ stimuli each developing substantial associative strength, whereas the A and B stimuli should have little strength. Consequently, an elemental model anticipates that $C$ and $D$ would summate if presented together, generating substantial responding to a $C D$ compound.

A simple application of the Pearce model, without assuming an important role of background stimuli, anticipates that the joint presentation of $C$ and $D$ should result not in summation but rather in less responding than that observed to $\mathrm{AD}$ and $\mathrm{BC}$. According to that theory, each of the $\mathrm{AD}$ and $\mathrm{BC}$ compounds acquires associative strength that generalizes to $C D$. The generalization that $C D$ receives from $\mathrm{AD}$ depends on the proportion of elements that they share, compared with those that are unique to each compound. However, the shared elements constitute only a fraction of each of the compounds, restricting generalization between them. More formally, the similarity of CD to $A D$ is determined by $p(D) /[p(D)+p(C)] * p(D) /$ $[p(D)+p(A)]$. If the elements are of equivalent potency, the computed similarity is .25 , meaning that $C D$ receives only a quarter of AD's strength. Similarly, $C D$ receives a quarter of BC's strength. The summed generalization from those two contributions falls far short of the strength of either $\mathrm{AD}$ or $\mathrm{BC}$, predicting less, rather than more, responding to the $C D$ compound. However, the prediction of the Pearce model is quite different if one assumes that the background stimuli are of a potency equivalent to those of the A, B, C, and D elements. Under those circumstances, responding to the $\mathrm{CD}$ compound is anticipated to be equal to that of the $\mathrm{AD}$ and $\mathrm{BC}$ compounds. The reason is that assuming a common $\mathrm{X}$ present in all of the compounds enhances the generalization among them and allows $\mathrm{CD}$ to benefit from more of the conditioning of the $\mathrm{AD}$ and $\mathrm{BC}$ compounds. However, even with this assumption of equal background potency, the theory does not anticipate summation in which responding to CD exceeds that to $\mathrm{AD}$ and $\mathrm{BC}$.

\section{Method}

\section{Subjects and Apparatus}

The apparatus was the same as that of Experiment 1. The subjects were 16 rats of the same origin and maintained in the same manner as those of Experiment 1.

\section{Procedure}

The animals were magazine trained with pellets in the manner of Experiment 1 . On each of the next 22 days, all animals received eight conditioning trials with each of three 30-sec compounds: $\mathrm{AB}$, $\mathrm{AD}$, and $\mathrm{BC}$. In all animals, the $\mathrm{AD}$ and $\mathrm{BC}$ compounds ended with the delivery of a pellet and the $\mathrm{AB}$ compound was nonreinforced. The $\mathrm{A}$ and $\mathrm{C}$ stimuli were the houselight (L) and flashing light (F), counterbalanced across animals; similarly the B and D stimuli were noise $(N)$ and tone $(T)$, counterbalanced in an orthogonal fashion. Trials were spaced with an ITI variable around a mean of $120 \mathrm{sec}$.

On the next day, all animals received an additional conditioning day, but four nonreinforced presentations of CD were intermixed during the last half of the session. Data from this session allow a comparison of responding to $C D$ with that to $A D$ and $B C$. On each of the next 2 days, the animals received another conditioning session. On the next day, an additional conditioning session was given but with one intermixed nonreinforced test presentation each of $\mathrm{C}$ and $\mathrm{D}$ and two of $\mathrm{CD}$. Data from this session allow a comparison of responding to $C D$ with that to $C$ and $D$.

\section{Results}

Acquisition proceeded smoothly. On the final day of training the mean responses per minute were $6.2,17.2$, 18.0 , and 9.4 during the pre-CS, $\mathrm{AD}, \mathrm{BC}$, and $\mathrm{AB}$ compounds, respectively. All animals showed greater responding to the reinforced $\mathrm{AD}$ and $\mathrm{BC}$ compounds than to the nonreinforced $\mathrm{AB}$ compound.

The data of most interest, from the initial test session presenting the CD compound, are shown in Figure 3. That figure displays the response rates during the pre-CS period and various compounds from the test that took

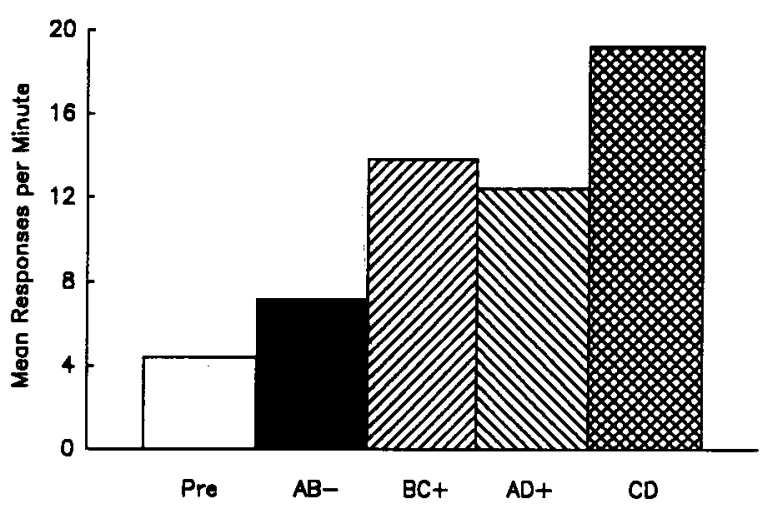

Figure 3. Results of the test session of Experiment 3. Responding is shown during the 30 -sec period prior to any stimulus, during the trained $\mathrm{AB}-, \mathrm{BC}+$, and $\mathrm{AD}+$ compounds, and during the tested $C D$ compound. 
place in the second half of Day 23 of conditioning. Responding during both the $\mathrm{AD}$ and $\mathrm{BC}$ compounds was greater than that during the $\mathrm{AB}$ compound $[T \mathrm{~s}(16)=0$, $p s<.01]$. Of more interest, responding during the tested $\mathrm{CD}$ compound exceeded that to both the AD and BC compounds $[T \mathrm{~s}(16)=14,15, p s<.01]$. Most importantly, responding to the CD compound exceeded that to whichever of those compounds was greater, determined on an individual animal basis $[T(16)=29, p<.05]$.

On the next day, responding was assessed to the individual $C$ and $D$ stimuli, as well as to the $C D$ compound. In that session, the mean responses per minute were 10.0 , 7.8 , and 17.8 to $\mathrm{C}, \mathrm{D}$, and $\mathrm{CD}$, respectively. Responding to the compound was superior to greater of the two elements, determined on an animal-by-animal basis $[T(16)=$ $10, p<.01]$.

\section{Discussion}

These results show clear evidence of summation in the responding to $\mathrm{CD}$ following an $\mathrm{AD}+, \mathrm{BC}+, \mathrm{AB}-$ conditioning procedure. Responding to $C D$ was more substantial than that either to the trained compounds or to its own elements. This result follows from such elemental theories as the Rescorla-Wagner model. But it is not anticipated from the Pearce version of a configural model, even under the assumption that the background stimulus has a potency equivalent to that of the stimuli being manipulated.

However, it does remain possible for a configural theory to predict these results if one is willing to assume a yet stronger background, one that is more potent than the elements. As indicated above, the more potent the background, relative to the elements, the greater the difference anticipated between $\mathrm{CD}$ and the trained compounds.

\section{EXPERIMENT 4}

The aim of this experiment was to examine another circumstance in which the Pearce configural theory anticipates that an equally salient background will be insufficient to produce summation. It also provided an opportunity to test the adequacy of assuming a yet stronger contribution of the background by examining further consequences of that assumption.

In this experiment two stimuli were conditioned but with different percentages of reinforcement. The A stimulus was followed by food on $100 \%$ of its occurrences whereas the B stimulus was followed by food on $50 \%$ of its occurrences. The question of interest was the level of responding that would be observed to a subsequently tested $\mathrm{AB}$ compound.

That question is of interest in its own right because it represents summation under conditions in which one of the stimuli has deliberately received less conditioning than the other. One might anticipate that under such circumstances the animal would display not summation but rather averaging of its response to A and B (see Weiss, 1972). Consequently, it is of some interest to ask whether a B stimulus that is weaker than an A stimulus will nevertheless lead to greater responding in the $\mathrm{AB}$ compound than to A alone.

The question takes on particular interest in the present context because the Pearce version of a configural theory fails to anticipate summation from the combination of two such stimuli. This is because the A stimulus experiences substantial generalization decrement when presented together with $B$, but the conditioned strength of the B stimulus is not sufficient to compensate for that decrement. This is most obvious in the case where no background stimulus is acknowledged, but remains true even under circumstances where the theory assumes that the background has a salience equivalent to that of the elements.

However, as in the case of the paradigm used in Experiment 2, a willingness to assume that the background has a potency greater than that of the elements does allow the theory to predict summation from the combination of a $50 \%$ stimulus and a $100 \%$ stimulus. As in that case, this results from the fact that the salient background enhances the similarity of both $A$ and $B$ to the $A B$ compound, reducing the generalization decrement and allowing greater use of their varied conditioned strengths.

Fortunately, that assumption has a further consequence in the present case that allows something of an independent assessment. The assumption of a highly potent $\mathrm{X}$ leads to the expectation not only of an enhanced generalization from $\mathrm{AX}$ and $\mathrm{BX}$ to $\mathrm{ABX}$, but also of an enhanced generalization between $\mathrm{AX}$ and $\mathrm{BX}$ themselves. The result is that when $\mathrm{AX}$ is reinforced on a $100 \%$ schedule and $\mathrm{BX}$ on a $50 \%$ schedule, much of the responding seen to BX is derived not from its own associative strength but rather from that it receives from $\mathrm{AX}$ by generalization. This heavy dependence on the conditioning of $\mathrm{AX}$ for responding to $\mathrm{BX}$ leads to the prediction that should AX undergo extinction, BX would undergo substantial decrement. Indeed, if one assumes that the $X$ is sufficiently potent to generate summation, then the Pearce theory predicts that extinction of AX would lead to complete abolition of responding to BX. Consequently, in this experiment a summation test of responding to $A B$ was followed by extinction to $A$ and retesting of $B$.

\section{Method}

\section{Subjects and Apparatus}

The apparatus consisted of eight chambers identical to those used in Experiment 1, with one exception. The light mounted on the ceiling of the chamber was moved to grid level outside the rear wall of the chamber; this light could be flashed at a rate of $1 / \mathrm{sec}$ to provide a flashing stimulus. The subjects were 16 rats of the same origin and maintained in the same manner as in Experiment 1.

\section{Procedure}

Magazine training. The animals received initial magazine training in the manner of Experiment 1.

Pavlovian conditioning. On each of the next 12 days, the animals received Pavlovian conditioning of $\mathrm{L}$ and $\mathrm{N}$. Each day contained sixteen 30 -sec presentations of each stimulus with an ITI that was variable around a mean of $120 \mathrm{sec}$. For half of the ani- 
mals, every presentation of $\mathrm{L}$ and a random $50 \%$ of the presentations of $\mathrm{N}$ terminated in a food pellet. For the other half of the animals, the reinforcement conditions were reversed.

Summation test. On the next day, the animals received a Pavlovian conditioning session, during the last half of which two nonreinforced presertations of the LN compound were given. These presentations were nonreinforced.

Extinction. On each of the next two sessions, the animals received extinction with the $100 \%$ stimulus. Each session contained 16 nonreinforced presentations of the appropriate stimulus.

Test. On each of the next two sessions, the animals received extinction with the $100 \%$ stimulus in the same manner; however, four nonreinforced presentations each of $\mathrm{L}$ and $\mathrm{N}$ were added to the end of the session.

\section{Results}

The results of the 12 acquisition sessions are shown in Figure 4. That figure displays the number of magazine entries during the $100 \%$ and $50 \%$ stimulus and during the 30 -sec pre-CS period. Because there were no systematic differences as a function of stimulus identity, the $\mathrm{L}$ and $\mathrm{N}$ stimuli have been combined according to the reinforcement likelihoods. It is clear that conditioning proceeded smoothly and that the terminal level of the $100 \%$ stimulus was substantially higher than that of the $50 \%$ stimulus $[T(16)=3.5, p<.01]$.

The results of the test period, during which presentations of the LN compound were intermixed with the continued conditioning of $\mathrm{L}$ and $\mathrm{N}$, are shown in Figure 5. That figure displays magazine responding during the pre-CS period, during the $100 \%$ and $50 \%$ stimuli, and during their joint presentation. In that test, responding to the pre-CS, $50 \%$, and $100 \%$ stimuli was similar to that observed at the end of training. Both stimuli produced more responding than that observed during the pre-CS period $[T \mathrm{~s}(16)=0, p<01]$. Moreover, responding was more substantial to the $100 \%$ than to the $50 \%$ stimulus $[T(16)=28, p<.05]$. Of most interest, responding was greater during the compound stimulus than that to either the $50 \%$ stimulus $[T(16)=0, p<.01]$ or the $100 \%$ stimulus $[T(16)=14, p<.01]$. Clearly, summation was observed.

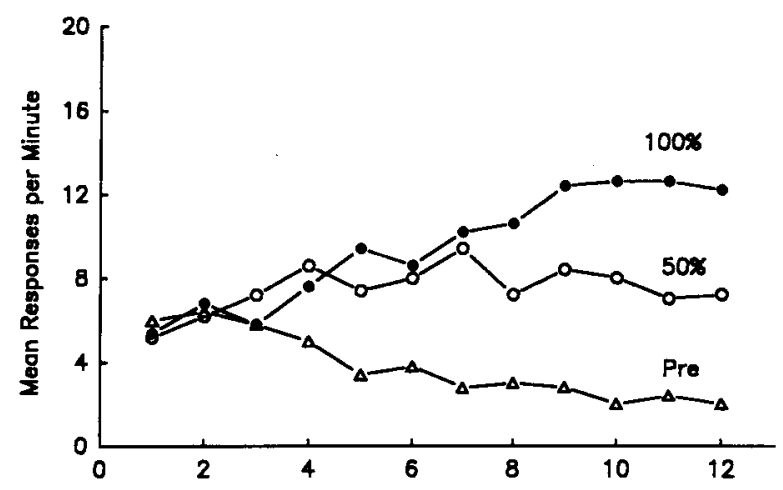

Figure 4. Acquisition of responding in Experiment 4. Responding is shown during the 30-sec period prior to any stimulus, and during the stimuli trained on $50 \%$ and $100 \%$ reinforcement schedules.

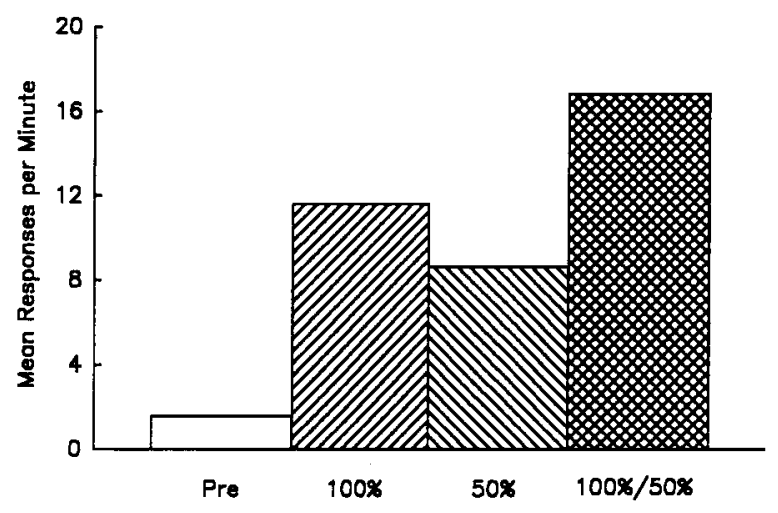

Figure 5. Test responding in Experiment 4. Responding is shown during the 30 -sec period prior to any stimulus, during the stimuli separately trained with $100 \%$ and $50 \%$ reinforcement, and during their compound presentation.

Over the next 2 days, responding to the $100 \%$ stimulus underwent deterioration with the institution of extinction. The results of the two test periods added on to the end of Extinction Sessions 3 and 4 are shown in Figure 6. That figure shows responding over the individual test trials for the $100 \%$ and $50 \%$ stimuli. It is clear that there was little performance to the extinguished $100 \%$ stimulus but substantially more to the $50 \%$ stimulus. That greater responding to the $50 \%$ stimulus was reliable on each of the test sessions $[T s(14)=10,5, p s<.05]$.

\section{Discussion}

These results show clear evidence of additive summation when a $50 \%$ reinforced and $100 \%$ reinforced stimulus are presented jointly. Despite the fact that it was of demonstrably lower associative strength, the $50 \%$ stimulus nevertheless was able to add to the strength of the $100 \%$ stimulus so as to boost responding. Although this result is anticipated by most elemental theories, it cannot be accommodated within the Pearce version of a configural theory in the absence of further assumptions. If one assumes that the background potency is less than that of the elements, then this theory anticipates that responding to the compound will be less than that to the $100 \%$ stimulus. To the degree that the background potency is assumed to exceed that of the individual stimuli, summation can be predicted. For instance, assuming that the background is four times as potent as the stimuli yields a net associative strength of about 1.25 for the compound, compared with that of 1.0 for the $100 \%$ stimulus.

However, the consequence of making such an assumption is that the theory anticipates substantial generalization between the $100 \%$ and $50 \%$ stimuli. As a result, it expects that extinction of the $100 \%$ stimulus will have a substantial negative impact on subsequently tested responding to the $50 \%$ stimulus. For instance, assuming that the background has four times the salience of the elements leads to the prediction that the $50 \%$ stimulus would lose all of its performance and become a net in- 


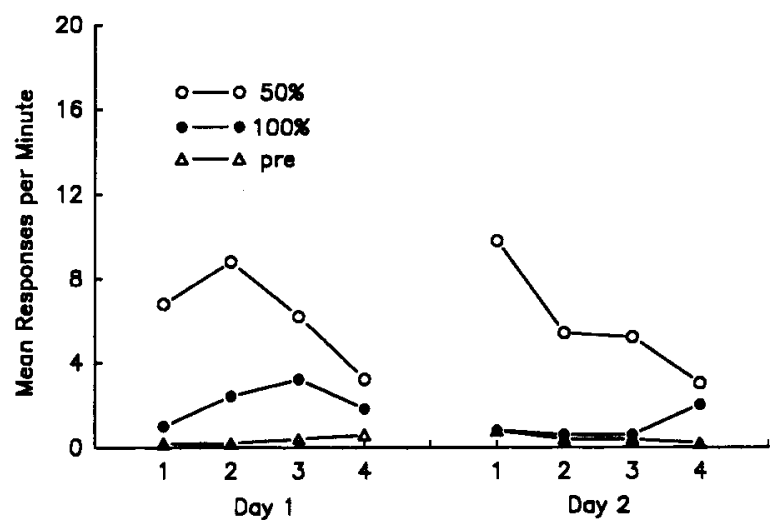

Figure 6. Responding following extinction of the $100 \%$ stimulus in Experiment 4. Responding is shown during the 30-sec period prior to any stimulus, and during the stimuli trained in $\mathbf{5 0 \%}$ and $100 \%$ reinforcement schedules.

hibitor well before the $100 \%$ stimulus becomes fully extinguished. The results shown in Figure 5 clearly contradict that prediction. The combination of substantial summation with continued performance to the $50 \%$ stimulus is inconsistent with the attempt of the Pearce theory to appeal to background stimulus salience to generate summation.

It should be noted, however, that this prediction from the Pearce model is not entirely free of the particular parametric assumptions that are made. The reason that summation between a $50 \%$ and a $100 \%$ stimulus fails is that $50 \%$ reinforcement is anticipated to yield a much smaller associative strength than the $100 \%$ stimulus. That in turn depends on the assumption that the rate parameter associated with nonreinforcement is not a good deal smaller than that associated with reinforcement. That assumption seems consistent with the markedly less responding seen to the $50 \%$ stimulus of the present experiment. However, assuming that the effect of nonreinforcement is much lower would give the model the ability to predict summation from a $50 \%$ and a $100 \%$ stimulus, at the price of anticipating much greater responding to the $50 \%$ stimulus.

\section{EXPERIMENT 5}

This experiment examined summation after yet another conditioning procedure in which the elements were deliberately made of unequal strength. Under this procedure, the animal received reinforcement following two compounds, $\mathrm{AB}$ and $\mathrm{CD}$; however, one element of one of those compounds was separately presented without reinforcement. The application of such an $\mathrm{AB}+, \mathrm{CD}+, \mathrm{A}-$ procedure should result in both $A B$ and $C D$ having substantial strength. This is arrived at over the course of training by $\mathrm{C}$ and $\mathrm{D}$ each developing moderate strength whereas $B$ becomes very strong and $A$ undergoes extinction. However, according to an elemental model, until A has become fully extinguished, it should be able to enter into summation. For instance, elemental models anticipate that prior to A's full extinction, A and C should summate, yield- ing greater responding to the $\mathrm{AC}$ compound than to either $\mathrm{A}$ or $\mathrm{C}$.

By contrast, a simple application of the Pearce model anticipates that the $\mathrm{AC}$ compound should be a weighted sum of the strengths of $A$ and $C$, hence yielding responding to $\mathrm{AC}$ that is less than that to $\mathrm{C}$ but greater than that to A. Adding the assumption of a highly potent background effectively shifts the strength of the $\mathrm{AC}$ compound closer to that of $\mathrm{C}$ and farther from that of $\mathrm{A}$. But even with the background as potent as the elements, responding to $\mathrm{AC}$ is anticipated to be less than that to $\mathrm{C}$ alone. Indeed, one has to assume that the background has approximately five times the potency of the discrete stimuli in order for the theory to anticipate that the responding to $\mathrm{AC}$ is similar to the responding to $\mathrm{C}$. Moreover, unlike the partial reinforcement procedure used to generate a relatively low level of strength to an element in Experiment 4, the present procedure yields the same predictions whatever the assumptions about the relative magnitudes of the rate parameters for reinforcement and nonreinforcement.

\section{Method}

\section{Subjects and Apparatus}

The apparatus was the same as that of Experiment 4. The subjects were of the same origin and maintained in the same manner as those of previous experiments.

\section{Procedure}

The animals were magazine trained with pellets in the manner of Experiment 1 . On each of the next 12 days, all animals received eight conditioning trials with each of three 30 -sec stimuli: $A B$, $C D$, and $A$. In all animals, the $A B$ and $C D$ compounds ended with the delivery of a pellet and the A stimulus was nonreinforced. For half the animals, the $\mathrm{AB}$ compound was composed of the $\mathrm{N}$ and the $\mathrm{F}$, whereas the $C D$ compound was composed of the $T$ and the $L$. In those animals, the noise played the role of $A$ and was separately nonreinforced. For the other half of the animals, the $A B$ and $C D$ compounds were interchanged, and $\mathrm{L}$ played the role of the separately nonreinforced stimulus. Trials were spaced with an ITI variable around a mean of $120 \mathrm{sec}$.

On the next day, all animals received an additional conditioning session, but four nonreinforced presentations each of $\mathrm{AC}$ and $C$ were added in intermixed fashion during the last half of the session. Consequently, during the second half of the session, the animals received four presentations each of $\mathrm{AB}+, \mathrm{CD}+, \mathrm{A}-, \mathrm{C}-$, and $A C$-. Because of the stimulus counterbalancing, $N$ played the role of $A$ in half the animals and $C$ in the other half; similarly, $L$ played the role of $\mathrm{C}$ in half the animals and $\mathrm{A}$ in the other half. Data from this session allow a comparison of responding to $\mathrm{AC}$ with that to the $\mathrm{A}$ and $\mathrm{C}$ elements.

\section{Results and Discussion}

Initial conditioning proceeded smoothly. On the final day, the mean responses per minute were $1.4,4.4,12.4$, and 12.2 for the pre-CS, $\mathrm{A}-, \mathrm{AB}+$, and $\mathrm{CD}+$ stimuli, respectively. All animals showed greater responding to the compounds than to both $A$ and the pre-CS period. Responding to A remained greater than that during the preCS period $[T(16)=3, p<.01]$.

The results of most interest, from the test period, are shown in Figure 7. That figure displays responding dur- 


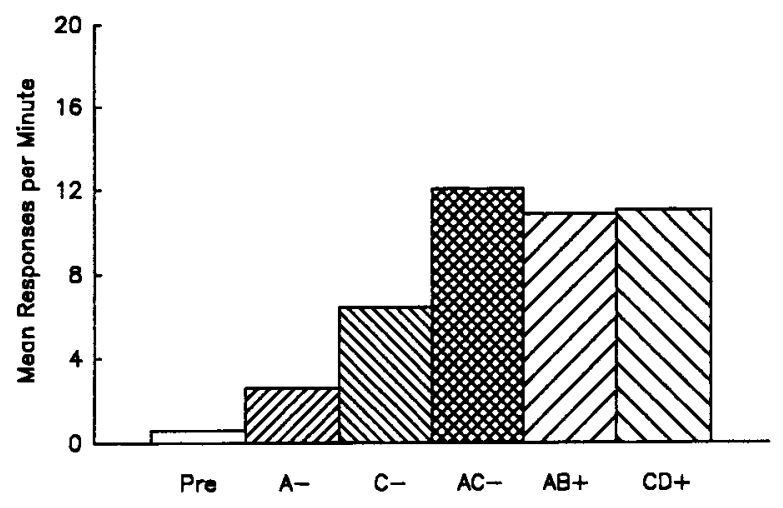

Figure 7. Test responding in Experiment 5. Responding is shown during the 30 -sec period prior to any stimulus, during the separately tested $\mathrm{A}, \mathrm{C}$, and $\mathrm{AC}$ stimuli, and during the previously reinforced $A B$ and $C D$ compounds.

ing the pre-CS period and during the $\mathrm{A}, \mathrm{C}, \mathrm{AC}, \mathrm{AB}$, and $\mathrm{CD}$ stimuli. Responding to the reinforced $\mathrm{AB}$ and $\mathrm{CD}$ compounds was high and comparable to that at the end of training. Similarly, responding to the previously nonreinforced A was low, but greater than that during the pre$\mathrm{CS}$ period. Responding to $\mathrm{C}$ was lower than that to the $\mathrm{CD}$ compound, presumably reflecting the effects of overshadowing by the $\mathrm{D}$ stimulus $[T(16)=6, p<.01]$. However, responding to $\mathrm{C}$ did exceed that to $\mathrm{A}[T(16)=5$, $p<.01]$.

Of most interest, responding to the $\mathrm{AC}$ compound was greater than that either to $\mathrm{A}[T(16)=0, p<.01]$ or to $\mathrm{C}$ $[T(16)=6, p<.01]$. Since $C$ generally produced greater responding than did $\mathrm{A}$, it is not surprising that responding to $\mathrm{AC}$ was also greater than that to larger of the two elements, determined on an individual animal basis $[T(16)=6, p<.01]$.

These results show clear evidence of summation between A and C. Despite the fact that A was treated in such a way that it yielded substantially less responding than did $\mathrm{C}$, nevertheless it was able to add to responding to $\mathrm{C}$ when they were presented in compound.

\section{GENERAL DISCUSSION}

The results obtained in the present experiments seem most compatible with elemental theories. Summation of associative strengths was obtained under fairly demanding conditions. Responding to a compound of separately trained elements was greater than that to a compound of other elements trained together. Responding to a compound was also greater than responding to the stronger of its own trained elements. That was true whether the stronger of the individual elements was determined on an animal-by-animal basis from equally treated elements (Experiments 1, 2, and 3) or was experimentally determined by the use of partial reinforcement (Experiment 4) or of separate nonreinforcement of an element (Experiment 5).
In the absence of further assumptions, the most popular configural model, that developed by Pearce, does not anticipate such summation. One natural avenue by which it might be modified to accommodate summation, assuming the contribution of a potent background, does have some attractions. For instance, assuming any nonzero salience of the background would yield summation in the simple summation design of Experiment 1. Moreover, it would provide a natural account of certain striking historical examples of summation, such as that reported by Reberg (1972). Reberg found that the joint presentation of two apparently fully extinguished fear elicitors yielded substantial levels of responding. Within the Pearce theory that assumes a potent background, this result is a straightforward consequence of the fact that a portion of the decrement in extinction would accrue to the background, rather than to the individual stimuli. Any given strength of inhibition thus conditioned to the background would be sufficient to prevent responding to single stimuli, which would retain some of their excitation, but be inadequate for overcoming their joint excitation.

In order for the Pearce theory to predict results like those observed in the present Experiments 3,4, and 5, the background must be assumed to be more salient than the discrete stimuli. Such an assumption would adequately explain the summation results of those experiments; however, it also leads to the expectation of substantial stimulus generalization between the stimuli themselves. The preservation of responding to the $50 \%$ stimulus after extinction of the $100 \%$ stimulus in Experiment 4 seems to disconfirm that prediction.

In general, introducing the notion of a salient background into the Pearce model can make a substantial difference in the kinds of predictions that it makes. This differs from the case of an elemental theory like the RescorlaWagner model, where assuming a salient background rarely makes a difference in the predicted asymptotic levels of performance. This is because in such a theory a nonreinforced background will ultimately approach zero associative strength, and zero strength stimuli make no contributions. Because assumptions about background stimuli in a model like that of Pearce can make such a difference, they have to be made with caution and evaluated in terms of independently measured consequences. At least in the case investigated here, such an evaluation makes the assumption much less attractive.

These data thus suggest that successful prediction of summation by a configural theory may not emerge from a consideration of background stimuli. Several other alternatives may be open to such a theory. For instance, some success can be had by explicitly acknowledging stimulus generalization among the elements of a compound. Assuming that the same modality elements of the present experiments share some elements will, in fact, allow this theory to accurately predict the results of Experiments 3 and 5 of the present report. However, it does little to help account for the results of Experiments 1, 2, and 4. Moreover, acknowledging stimulus generalization among the 
elements leads this model in general to anticipate greater summation when the components of a compound are drawn from the same, rather than different, stimulus modalities. That prediction seems inconsistent both with available published results in autoshaping (Rescorla \& Coldwell, 1995) and eyelid conditioning (Kehoe, Horne, Horne, \& Macrae, 1994) and with unpublished data from our laboratory using the present preparation.

Another alternative would be to modify much more basic assumptions, such as the nature of the generalization rule. As noted previously, existing configural and elemental models can be thought of as points on a continuum in terms of the degree to which an $\mathrm{AB}$ compound receives generalization from its separately conditioned elements (see Kehoe, 1988). Elemental models assume complete generalization, whereas configural models, such as that proposed by Pearce, anticipate substantially less generalization. It remains to be seen whether one could develop an intermediate generalization rule that permits summation under circumstances like that explored here but that also preserves the other attractions of a configural model.

\section{REFERENCES}

Aydin, A., \& Pearce, J. M. (1995). Summation in autoshaping with short- and long-duration stimuli. Quarterly Journal of Experimental Psychology, 48B, 215-234.

Colwill, R. M., \& Rescorla, R. A. (1985). Post-conditioning devaluation of a reinforcer affects instrumental responding. Journal of Experimental Psychology: Animal Behavior Processes, 11, 120132.

Forbes, D. T., \& Holland, P. C. (1985). Spontaneous configuring in conditioned flavor aversion. Journal of Experimental Psychology: Animal Behavior Processes, 11, 224-240.

Hull, C. L. (1943). Principles of behavior. New York: AppletonCentury-Crofts.
KEHOE, E. J. (1986). Summation and configuration in conditioning of the rabbit's nictitating membrane response. Journal of Experimental Psychology: Animal Behavior Processes, 12, 186-195.

KEHOE, E. J. (1988). A layered network model of associative learning: Learning-to-learn and configuration. Psychological Review, 95, 411-433.

Kehoe, E. J., Horne, A. J., Horne, P. S., \& Macrae, M. (1994). Summation and configuration between and within sensory modalities in classical conditioning of the rabbit. Animal Learning \& Behavior, 22, 19-26.

Pavlov, I. P. (1927). Conditioned reflexes (G. V. Anrep, Trans.). Oxford: Oxford University Press.

Pearce, J. M. (1987). A model of stimulus generalization for Pavlovian conditioning. Psychological Review, 94, 61-73.

Pearce, J. M. (1994). Similarity and discrimination: A selective review and a connectionist model. Psychological Review, 101, 587607.

REBERG, D. (1972). Compound tests for excitation in early acquisition and after prolonged extinction of conditioned suppression. Learning \& Motivation, 3, 246-258.

Rescorla, R. A., \& Coldwell, S. E. (1995). Summation in autoshaping. Animal Learning \& Behavior, 23, 314-326.

RESCORLA, R. A., \& WAGNER, A. R. (1972). A theory of Pavlovian conditioning: Variations in the effectiveness of reinforcement and nonreinforcement. In A. H. Black \& W. F. Prokasy (Eds.), Classical conditioning II: Current research and theory (pp. 64-99). New York: Appleton-Century-Crofts.

Wagner, A. R., \& Rescorla, R. A. (1972). Inhibition in Pavlovian conditioning: Application of a theory. In R. A. Boakes \& M. S. Halliday (Eds.), Inhibition and learning (pp. 301-336). New York: Academic Press.

WEISs, S. J. (1972). Stimulus compounding in free-operant and classical conditioning: A review and analysis. Psychological Bulletin, 78, 189-208.

Whitlow, J. W., JR., \& WAGNeR, A. R. (1972). Negative patterning classical conditioning: Summation of response tendencies to isolable and configural components. Psychonomic Science, 27, 299-301.

(Manuscript received April 4, 1996; revision accepted for publication July 12, 1996.) 